Review Article

Open Access

\title{
Microbiome: pharmacokinetics, pharmacodynamics and drug/xenobiotic interactions
}

\author{
Ojezele, M. O. \\ Department of Pharmacology \& Therapeutics, Delta State University, Nigeria \\ Correspondence to: matlar2002@gmail.com, +2348033923332
}

\begin{abstract}
:
The participation of microbiota in myriads of physiological, metabolic, genetic and immunological processes shows that they are a fundamental part of human existence and health maintenance. The efficiency of drugs' absorption depends on solubility, stability, permeability and metabolic enzymes produced by the body and gut microbiota. Two major types of microbiota-drug interaction have been identified; direct and indirect. The use of antibiotics is a direct means of targeting intestinal microbes and short-term use of antibiotic can significantly alter the microbiome composition. It is noteworthy that not every microbial drug metabolism is of benefit to the host as some drugs can shut down microbial processes as observed in the co-administration of antiviral sorivudine with fluoropyridimide resulting in a toxic buildup of fluoropyridimide metabolites from blockade of host fluoropyridimide by the microbialsorivudine metabolite. It has been reported that many classes of drugs and xenobiotics modify the gut microbiome composition which may be detrimental to human health. Microbiome-drug interaction may be beneficial or detrimental resulting in either treatment success or failure which is largely dependent on factors such as microbial enzymes, chemical composition of candidate drug, host immunity and the complex relationship that exists with the microbiome. The effects of microbiota on pharmacology of drugs and vice versa are discussed in this review.
\end{abstract}

Keywords: microbiome; pharmacokinetic, pharmacodynamic, drug, xenobiotic

Received September 27, 2019; Revised November 30, 2019; Accepted December 3, 2019

Copyright 2020 AJCEM Open Access. This article is licensed and distributed under the terms of the Creative Commons Attrition 4.0 International License (http://creativecommmons.org/licenses/by/4.0), which permits unrestricted use, distribution and reproduction in any medium, provided credit is given to the original author(s) and the source.

\section{Microbiome: pharmacocinétique, pharmacodynamique et interactions médicamenteuses/xénobiotiques}

\author{
Ojezele, M. O.
}

Département de pharmacologie et de thérapeutique, Université d'État de Delta, Nigeria

Correspondance à: matlar2002@gmail.com; +2348033923332

\begin{abstract}
Abstrait:
La participation du microbiote à des myriades de processus physiologiques, métaboliques, génétiques et immunologiques montre qu'ils sont un élément fondamental de l'existence et du maintien de la santé de l'être humain. L'efficacité de l'absorption des médicaments dépend de la solubilité, de la stabilité, de la perméabilité et des enzymes métaboliques produites par le corps et le microbiote intestinal. Deux types principaux d'interaction microbiote-médicament ont été identifiés; direct et indirect. L'utilisation d'antibiotiques est un moyen direct de cibler les microbes intestinaux et une utilisation à court terme d'antibiotique peut modifier de manière significative la composition du microbiome. Il est à noter que tous les métabolismes de médicaments microbiens ne sont pas bénéfiques pour l'hôte, car certains médicaments peuvent arrêter les processus microbiens observés lors de l'administration concomitante d'antiviral sorivudine et de fluoropyridimide, ce qui entraîne une accumulation toxique de métabolites de fluoropyridimide résultant du blocage du fluoropyridimide par l'hôte. métabolite microbien-sorivudine. Il a été rapporté que de nombreuses classes de médicaments et de xénobiotiques modifiaient la composition du microbiome intestinal, ce qui pourrait nuire à la santé humaine. Une interaction médicamenteuse-microbiome peut être bénéfique ou préjudiciable, entraînant le succès ou l'échec du traitement,
\end{abstract}


qui dépend en grande partie de facteurs tels que les enzymes microbiennes, la composition chimique du médicament candidat, l'immunité de l'hôte et la relation complexe qui existe avec le microbiome. Les effets du microbiote sur la pharmacologie des médicaments et inversement sont discutés dans cette revue.

Mots-clés: microbiome; pharmacocinétique, pharmacodynamique, médicament, xénobiotique

\section{Introduction:}

The understanding of the facts that microbiota/microbiome play an important role in the lives of humans dictates how much microorganisms matter. Their participation in myriads of physiological, metabolic, genetic, and immunological processes shows that they are a fundamental part of humans' existence and health maintenance. The customized genetic differences in the microorganisms exceeding the human genome also have significant consequences for drug reactions in humans. This is as a result of microbiota involvement in its metabolism through the action of enzymes and a complex hostmicrobiota interaction as the host genome can influence the microbiome in terms of phenotypes and function $(1,2)$.

Most drugs are designed for oral administration. Drugs movement through the gut makes them come in direct contact with other extra-intestinal organ such as the liver. Also, they encounter intestinal environmental conditions such as the stomach acids as well as coming in contact with myriads of gut microorganisms from small and large intestines. The efficiency of drugs' absorption depends on solubility, stability, permeability and metabolic enzymes produced by the body and gut microbiota $(3,4)$. Furthermore, parenterally administered drugs, as well as their metabolites, also reach the intestines through biliary secretion making the gut a central station for drug metabolism prior to absorption thereby the bioavailability and distribution of the drug is altered $(1,3)$. However, little is known about microbiome and drug interactions $(4,5)$.

Two major types of microbiota-drug interaction have been identified; direct and indirect.Indirect interaction may involve competition between microbiota-derived metabolites and administered drugs for the same host metabolizing enzyme; microbiome reactivation of secreted inactive drug metabolite; effect on the immune system and effect on the overall level of the metabolizing enzymes in the liver and intestine. Direct interactions include a partial or complete biochemical transformation of a candidate drug into more or less active metabolites by microbially-derived enzymes known as
Microbiome-Derived Metabolism (MDM) (4). Although MDM has been observed about 50 years ago it was overlooked in drug development. Studies that are investigating this process focused on specific bacterium against a specific drug rather than a systematic assessment of human gut microbiome's capability to metabolize several oral drugs. This problem is due to the microbiome's huge complexity and the overwhelming technical challenge of testing several drugs against several isolate cultures under multiple conditions. More so, the unavailability of the global standard has hampered the reliability to predict undesired microbiome effect on pharmacodynamics and pharmacokinetics of drugs (4).

\section{Methodology:}

A review of microbioata as it relates to pharmacology of drugs was conducted. The materials used for the review included relevant published articles in journals spanning the period 2001 to 2019, using google and google scholar search engines. The keywords used for the search were 'xenobiotic', 'microbiome', 'microbiota', 'pharmacology' and 'gut microbioata'. A total of 150 articles were initially identified but following assessment, 53 were selected for the review.

\section{Microbiome effects on drugs}

The use of antibiotics is a direct means of targeting intestinal microbes and short-term use of the antibiotic drug is enough to significantly alter the microbiome composition (6). A population-based study involving deep sequencing of gut microbiomes of 1135 subjects showed an association between microbial species and 19 drug groups (7). The degree to which the gut microbiome is affected may depend on the type and number of drug taken either combined or singly by an individual. While drugs such as Non-Steroidal Anti-inflammatory Drugs (NSAIDs), and Proton Pump Inhibitors (PPIs) showed differences in the individual gut microbiome composition, drugs such as PPIs, antidepressants and antipsychotics showed significant association with single bacteria abundance $(6,8)$. Conversely, in a study, gliclazide (antidiabetic) pre-treated with probiotics showed 
higher blood levels of gliclazide in treated rats compared with non-treated rats revealing that gut microbiota may mediate the degree to which a drug is absorbed (9). Furthermore, another study reported a 3-day administration of Lactobacillus reuteri (K8) to have reduced orally administered acetaminophen in mice while another variant of the same species (K9) showed no observable effect; this effect may be mediated by probiotic-induced-modulation of the gut microbial enzyme activity as sulfatase and arylsulfate transferase were increased by the probiotic and $\beta$-glucuronidase enzyme decreased (10).

\section{Microbiome modifications of drug absorption}

The bioavailability and absorption rate of candidate drugs administered orally pose a major obstruction to drug efficiency as about $40 \%$ of these oral drugs are no more in use as a result of poor bioavailability and/or pharmacokinetics in the 1990s (11). The governing principles of absorption totally lies in the chemical properties of the drug and how the host can actively import or export the molecule. Early studies implied that drug transport may be influenced by microbiome in a composition-dependent manner, and multiple animal studies also demonstrated that the microbiome modulates absorption of drugs (2).

Among the various methods of invitro screening of drug absorption parameters, cellbased drug absorption assays are one of the most common and readily adopted for highthroughput screening (12). The Caco-2 cell line (ATCC HTB-37) is an ideal model for these assay types as it has the ability to differentiate into a monolayer homologous to the enterocytes of the small intestines with characteristic expression of transporters which include $p$ glycoprotein and OATP-A/B $(2,12)$. When the Caco- 2 cells are grown on a semi-permeable membrane such as the Transwells, a polarized membrane is produced with an apical and basolateral chamber which is functionally homologous to the intestinal lumen and underlying circulation respectively. The apical to basolateral and vice versa are measured for a candidate drug, or suitable surrogate compounds such as p-glycoprotein model substrates digoxin, calcein-AM, or rhodamine 123 (13, 14). Complex Caco-2 cell models such as the gut-on-a-chip and HuMiX models allow for 3D structures development including the villi, and divergent cell types with a relative enhancement of CYP3A4 activity while facilitating co-culture with bacterial cultures (2).
Several modes through which the microbiome regulatess host transport have been suggested, but they either include controlling host gene expression, Ilosteric regulators, substrate competition, or binding of microbial products acting as inhibitors (2). Through the use of quantitative reverse transcriptase polymerase chain reaction ( $q R T$ PCR) panels or RNA sequencing, examination of host expression can be achieved in a targeted manner. Meanwhile, the examination of co-differentially regulated genes may give more insight into the pathways through which modulation occurs (2). For instance, pglycoprotein is under the control of FXR, PXR, AP-1 and NFKB pathways, but the examination of co-regulated transcripts employing the pathway analysis tools may help detect the active specific pathway. It has been established that indole metabolites and other microbial-derived compounds function as PXR ligands $(15,16)$.

The intestinal lumen contains a variety of cell types, many of which do not have an absorptive function. To be able to target absorptive enterocytes therefore, laser capture microdissection or single-cell RNA sequencing may be employed to limit differential expression analyses to target cell types of interests. This may be essential in the case of genes which have functions in other cell types such is the case of P-glycoprotein expressed in phagocytes and T cells (17).

\section{Microbiome-drug metabolism}

It is noteworthy that not every microbial drug metabolism is of benefit to the host as some drugs can shut down microbial processes as observed in the co-administration of antiviral sorivudine with fluoropyridimide resulting in a toxic build up of fluoropyridimide metabolites as a result of blockade of host fluoropyridimide by the microbial-sorivudine metabolite (2). About fifty known drugs have been shown to be metabolized by bacteria, however neither the microbial species nor the genetic determinants responsible for these drug metabolisms are known (18). Moreso, since there are no observable pieces of evidence for bacterial metabolism to have taken place, the number of drugs metabolized by the microbiome is likely to be higher (19).

The discovery of high-throughput culturing has made screen collections and bacteria selection possible, representing extensive panels of strains for drug metabolism that are human-associated. It is possible to observe substrate loss or new metabolite production by the co-incubation of 
each microbial strain or collection (synthetic community) with each of the desired drugs. While high-throughput isolate screens have the merit of detecting bacteria capable of drug metabolism with direct interpretation, highdensity plate-based culturing and robotics provide a superficial sampling of microbial genetic variation as development of analytical method for the drug is a primary limitation (2). Large chunk of the genetic variations within a species is achieved via horizontal gene transfer which is usually associated with resistance to antibiotics $(2,20)$.

Many phenotypes such as the ability to inactivate the drug digoxin, a cardiac drug, occurs periodically within species (21). The presumptive selection of representative strains may likely not have the genes of interest due to superficial sampling at the species level; however, this approach has proven successful in the identification of multiple drug metabolizing bacterial species (22). The use of ex vivo human stool samples incubations may be an alternative to culture-collection screens as this method has the advantage of identifying many of the drugs that are susceptible to microbial metabolism. Nonetheless, inter-strain antagonism may obscure metabolism when testing a community of microorganisms collectively and this may be true especially if these metabolizing microbes are impeded by antimicrobial products such as bacteriocins or organic acids of metabolically inactive strains (23). In addition, using media formulations, some microbes may be favored, conferring dominance on them over the entire culture. One method to overcome this is to perform ex vivo incubations through manifold media formulations with broad selection pressures ready for aerobic spore-forming Gram-positive, strictly anaerobic, and Gram-negative microbes (2).

Another methodology for investigating microbial biochemical changes from a mixed community is the use of faecalase preparation, which is a cell-free faeces extract including microbial enzymes (2). This method has been used to assess gut microbial enzyme activity against herbal glycosides generating genotoxic aglycone products (24). Also, this method has been used to demonstrate that lovastatin, a cholesterol-lowering drug can be metabolized by the gut bacteria (25). However, the presence of, or competition for, essential cofactors and membrane-bound coenzymes may lead to false-negative results in this methodology; these may or may not be present in the faecalase preparation. Furthermore, the secretion of cytosolic enzymes which may not be active in buffer conditions and the lack of constant generation of $N A D(P) / F A D$ in the cell may inhibit metabolic activity and consequently, the sensitivity of this methodology can be problematic to define (21).

A number of approaches may be employed to unearth the molecular mechanisms responsible for detecting metabolism. Since it has been established that a specific microbial strain is capable of metabolism, multi-omics can be especially informative (2). In the bacterial genome, genes that are functionally related are typically restricted and transcriptionally regulated as operons. After stimulated by a ligand, there is the induction of specific effector gene expression and this can be exploited for the identification of candidate effector enzymes and molecules through gene expression analysis such as RNA sequencing (RNA-seq) by differentiating expression between drug-exposed and vehicle controls (2). However, not all enzymes are subject to substrate-mediated transcriptional regulation and there is need for caution to syncronize the the drug-exposed cultures and growth phase of control prevent false-positive results stemming from changes in cellular physiology (19). Lastly, many non-antibiotic drugs may have either bactericidal or bacteriostatic effects invitro, potentially causing serious changes in gene expression due to stress responses. As soon as specific genes are identified, their induction can be readily confirmed by quantitative reverse transcriptase PCR (qRT-PCR) on independent cultures. Nonetheless, followup studies are very paramount to make causal links between the identified genes and specific biochemical reactions (2). Nuclear receptors especially CAR, PXR, FXR and AHR regulate the expression levels of transporters and enzymes for drug-metabolizing $(26,27)$. The CAR, PXR and FXR are classified members of the steroid receptor superfamily that regulate their associated genes through the formation of a DNA-binding heterodimer with retinoid $X$ receptor (27). The CAR receptor regulates the following drug-metabolizing enzymes; CYP2B6 and CYP2C9 (phase 1), UGTB1 and SULT1E1 (phase 2) and organic anionic transport protein OATP1B3 (phase 3). Meanwhile PXR regulates CYP3A, CYP3B, CYP2B, CYP2C and GSTA1, UGT1A3, as well as UGT1A6 in phase 1 and phase 2 metabolizing enzymes respectively (27). Both CAR and PXR regulate overlapping groups of genes involved in phase 1, 2 and 3 metabolism (27). 


\section{Impacts of xenobiotics and drugs on gut microbiome}

It has been reported that many classes of drugs and xenobiotics modify the composition of gut microbiome which may be detrimental to human health. Infection by Clostridium difficile has been reported to be associated with the use of proton pump inhibitors by patients (28). Several drugs such as sulfasalazine, antibiotics, digoxin and phenacetin have been investigated to have the greatest impact on the functionality of gut microbiome but the degree to which antibiotic treatment modulates the metabolism of orally administered drugs is subject to further perusal (27). Additionally, many xenobiotics have been reported to modify the gut microbiota and most notable xenobiotic is arsenic which has been demonstrated in a mouse model to significantly decrease the abundance of Firmicutes which are mainly producers of butyrate thereby altering the composition of indole and glucuronide metabolites (27).

\section{Microbiome impacts on phytochemical metabolism and bioavailability}

The most notable function of hostmicrobial co-metabolism is the conversion of dietary plant substances into bioactive molecules. This role has attained accelerated importance with traditional medicine and herbal supplements now widespread (29). Phenolics and flavonoids are the most common phytochemicals most disposed in the human colon to microbial metabolism (27). More over, microbiome impact on phytochemicals includes metabolic changes involving esterases, demethylations, glycosidases, dehydroxylations and decarboxylations. Curcumin is the beststudied naturally occurring phenolics due to its anti-oxidant and anti-inflammatory activities. Its pharmacologic activity is thought to be as a result of tetrahydrocurcumin, a metabolite produced by gut microbiota. Escherichia coli from human faeces exhibits the highest curcumin metabolizing activities $(27,30)$.

\section{Microbiome impacts on drug/xenobiotic metabolism and pharmacokinetics}

The gut microbiome uses several mechanisms to modify the nature, potency and toxicity of both drugs and xenobiotics. These mechanisms are achieved through; (i) expression of enzymes that either inactivate or activate drugs metabolically, seen in the case of sulfasalazine for the treatment of gut inflammation being converted to 5-amino 5salicylic acid (its active form) by microbial enzymes while on the other hand, digoxin is being inactivated by a cardiac glycoside produced by the bacterium Eggerthella lenta; (ii) the drug may be separated in order to store its component by binding directly to the bacterial organism as in the case of L-DOPA sequestered by Helicobacter pylori; (iii) the drug may be reactivated metabolically by expressed enzymes produced by microbes and an example of this mechanism is irinotecan (CPT-11), a drug that was previously inactivated in the liver via glucuronidation, being reactivated by bacterially expressed betaglucuronidase resulting in diarrhoea within the intestines; (iv) metabolites that function as intermediates may be generated by the microbiota and an example is the toxicity in melamine as a result of the microbial formation of cyanuric acid (its metabolite); and ( $v$ ) microbial $\mathrm{p}$-cresol and metabolites produced by the host from a candidate drug acetaminophen may directly compete for a host enzyme SULT1A1 $(29,31,32)$. Metabolic reactions of microbiota known to largely affect the bioactivity of xenobiotics and drugs comprise reduction, proteolysis, hydrolysis, acetylation, dihydroxylation, deacetylation, deconjugation and deglycosylation processes (27).

Several enzymes expressed by gut microbes have been identified. These include azoreductases which have been reported to metabolize the drug prontosil and neoprontosil. When injected intravenously, the drugs gain entry into the intestine and are converted to sulfanilamide by gut microbiota. Another drug, balsalazide is metabolized into 5-aminosalicylic acid by microbially expressed enzymes (azoreductases). However, antibiotic treatment suppresses the conversion of orally administered balsalazide into 5-aminosalicylic acid, prontosil and neoprontosil to sulfanilamide in rat respectively (3).

Among many studies that provide insight into the upcoming role of gut microbiome in the metabolism and pharmacokinetics of drugs, bacterial nitroreduction reactions are of keen interest as they can largely affect the pharmacologic activity of nitroaromatic drugs such as chloramphenicol, 2-chloro-5-nitro-N-phenoxybenzamine (GW9662), nitrobenzodiazepine and 5-(aziridine-1-y1)2,4-dinitrobenzamide (CB1954) (27). Chloramphenicol, a substrate of bacterial nitroreductase, GW9662 is an antagonist of peroxisome proliferator which is activated by receptor $\mathrm{g}$ and a chemo-preventive agent, while the principal plasma metabolite of GW996 is an amine metabolite. The nitroreduction of this aimine metabolite by bacterial nitroreductases 
can largely change its mutagenicity. The study of both nitroreduction of nitrobenzodiazepine (an addictive sedative in the treatment of anxiety and sleep disorders) resulted in further characterization of bacterial nitroreductases and its metabolism (27).

Nitroreductase being identified as NfsB is expressed by $E$. coli and it has been suggested that NfsB may be suitable for development of anti-addictive agents as nitroreduction leads to the inactivation of nitrobenzodiazepine. CB1954, an anticancer drug which is a dinitrobenzamide prodrug has been developed to target cancer cell through the delivery of NfsB transgene (33). Furthermore, other enzymes expressed by $E$. coli species capable of nitro and azo reductions under aerobic conditions comprise NfsA and AzoR. Other organisms capable of expressing nitroreductases include Bacillus spp., Mycobacterium, Enterobacter and Staphylococcus (34). Although nitroreductases are involved in antibiotic resistance, their role in the metabolism of currently prescribed drugs is yet undetermined.

$\mathrm{N}$-oxide reduction by bacteria lies at the central relationship between microbial enzymes and the host in the metabolism of an inhibitor (BILR355) of the human immunodeficiency virus (HIV). BILR355 is metabolized by CYP3A, nonetheless, a study in which simultaneous administration of BILR355 with ritonavir occurred, unravelled a distinct role of aldehyde oxidase and gut bacteria (35). The biotransformation of BILR355 involves a twostep process; the first step involves the reduced form of $\mathrm{N}$-oxide generated by the gut bacteria while the second step involves bacterially derived metabolite subject to the further host enzymes metabolism either by CYP3A or aldehyde oxidase. Nevertheless, the action of CYP3A is inhibited while the bacterial enzyme or aldehyde oxidase takes over (27, 35).

The relationship between the gut bacterial enzymes and host cytochrome P450 is also required in fostamatinib (tyrosine kinase inhibitor) metabolism. Fostamatinib is a prodrug oxidatively metabolized by CYP3A4 upon cleavage of alkaline phosphatases. Furthermore, a metabolite believed to have been formed in faeces via O-demethylation and dihydroxylation by gut anaerobes has been recognized. An invitro colon model supported with metabolomics has shown that cholesterol dissolving drug, simvastatin can be metabolized by the colon microbiota where anaerobes are prevalent. Simvastatin, designed to inhibit 3-hydroxyl-3methylglutaryl coenzy- me $A$ in the liver to reduce cholesterol level is hydroxylated and subjected to b-oxidation, glutathione conjugation, and glucuronidation $(36,37)$. Other drugs metabolized by the colon anaerobic bacteria include ranitidine $\left(\mathrm{H}_{2}\right.$ antagonist) and prednisolone, a glucocorticoid agonist and anti-inflammatory agent (27).

Arsenic and polyaromatic hydrocarbons are xenobiotics that have been subjected to microbial metabolism in the gut (27). A study using a simulator of the microbiota (human gut) showed that colon microbiota can be involved in the extensive metabolism of arsenic (38). Sulphate reducing bacteria such as the Desulfovibrio desulfuricans are notable for their $\mathrm{H}_{2} \mathrm{~S}$ production which converts monomethyl arsenic acid to monomethyl monothioarsenic which is a more noxious form of arsenic (38). The metabolism of polyaromatic hydrocarbons involves oxidation reactions by the cytochrome P450, followed by phase 2 conjugation with either glucuronic acid gluthathione or sulphate. Report from the simulation of human microbiota shows that colonic human bacteria are also capable of biotransforming polyaromatic compounds such as naphthalene, phenanthrene, pyrene, and benzo[a]pyrene. Micrococcus luteus has been identified to metabolize benzo[a]pyrene and the most likely enzyme involved was DszA/NtaA-like oxygennase $(27,39)$.

\section{Microbiome, detoxification and excretion}

Detoxification and excretion of drugs in the human system are intrinsic to drug distribution and are basically propelled by hepatocytes for most medications (2). The proximity of the liver to the gut makes it close relatives linked by the biliary tract and the portal vein allowing for metabolite exchange derived by microbes and host as well as other compounds (2). There are evidences pointing to the gut-liver axis in which the microbiome and the liver interact through biliary excretion, recycling, signalling and regulation of gene expression (40). Furthermore, evidence also point to the gut-liver axis in relation to drug excretion. A study on differential expression of xenobiotic metabolism in the livers of germfree or conventionally raised mice suggested that microbial metabolites of tryptophan act as aryl hydrocarbon receptor (AHR) activators (2, 41).

\section{Microbiome and drug interactions in anticancer therapies}

Anticancer drugs are specially designed to target malignant cells but sometimes not without being toxic to the host cells which may 
result in life-threatening adverse effects. Anticancer drugs are also faced with challenge of resistance which is a major cause of chemotherapeutic treatment failure in human tumours and this failure can be partially due to genetic factors. However, efforts have been channelled into the development of more specific anticancer therapeutic approaches with lesser host cell toxicity. Immunotherapy has been a new paradigm in oncology where drugs are targeting immune cells rather than cancer cells, that is, the drugs are aimed at stimulating the host antitumor immune response (42).

The microbiome can interfere with the efficacy of anticancer treatment by metabolizing the drugs, inactivating or activating them. Also, they can modulate the immune system, interfere with side effects or the therapy modulates the microbiome (43). Both gut microbiota and intra-tumour bacteria can modulate chemotherapy and mediate its toxic effects. Mycoplasma infections, especially from Mycoplasma hominis and its presence in tumour tissues, have been reported in some types of cancers $(44,45,46)$ as they are found to express nucleoside analogue-catabolizing enzymes that impairs drug efficacy (44). In a study involving simultaneous injection of $M$. hominis, infected colon cancer cells showed resistance to gemcitabine $\left(2^{\prime}, 2^{\prime}\right.$-difluorodeoxycytidine) due to deamination to its inactive metabolite $\left(2^{\prime}, 2^{\prime}\right.$-difluorodeoxyuridine) in the liver (46). Other organisms, especially Gammaproteobacteria were reported to also induce gemcitabine resistance due to the expression of a bacterial long-form enzyme, cytidine deaminase. In a colon cancer mouse model, resistance to gemcitabine-induced by Gammaproteobacteria was reversed by coadministration of ciprofloxacin, hence confirming the role of these bacteria in anticancer treatment failure $(41,46)$. Invivo studies showed that $E$. coli impairs the action of gemcitabine as shown by increased tumour volume and reduced survival. Meanwhile, cytotoxicity of the drug, CB1954, was increased by the action of nitroreductase. Evidence that bacteria may influence the value of chemotherapy regimen, decreasing certain drug activities while enhancing the activity of others $(42,45)$ was observed in microbiota response for platinum compounds where mice dosed with combination of cisplatin and Lactobacillus showed better treatment response. These perceived effects were associated with the modulation of VEGFA, BAX and CDKN1B genes expression in the tumour and to the bacterial enhancement of the $\mathrm{T}$ cell immunity (47). Furthermore, cyclophosphamide (CTX) treatment in tumour-bearing mice caused translocation of Gram-positive bacteria such as Lactobacillus johnsonii, Lactobacillus plantarum, Lactobacillus murinus, Barnesiella intestinihominis and Enterococcus hirae into the mesenteric lymph node and spleen where they were involved in stimulation of Th1 and Th17 immune response. Germ-free mice treated with antibiotics against Gram-positive bacteria failed to generate this immune response and induced resistance to CTX in the study (43, 48).

Selective Estrogen Receptor Modulators (SERMs) such as tamoxifen can modulate microbiome composition as they can be toxic for organisms such as Pseudomonas aeruginosa, Klebsiella pneumoniae, Acinetobacter baumannii, Porphyromonas gingivalis, Streptococcus mutans, Enterococcus faecium and Bacillus stearothermophilus. No bacterial drug metabolism has been related to SERMs, but resistance to tamoxifen has been shown to be a reason for changes to cancer cell metabolism modulated by the microbiome. Furthermore, tranxes may be subject to microbial metabolism and interfere with bacterial LPS in activating the immune system (43). Poly ADP ribose polymerase (PARP) inhibitors are potential drugs in the future treatment of breast cancer as they have been shown to increase the diversity of gut microbiome. There has been no report on the relationship between aromatase inhibitors, navelbine, GnRH-analogs and the microbiome (43).

\section{Microbiome and anti-hypertension drugs}

A study examined 52 common drugs of which 5 were anti-hypertension medications ( $\beta$-blockers, a-blockers, ACE inhibitors, Sartan, and calcium channel blockers) and the microbiota (5). $\beta$-blockers in relation to Firmicutes and a-blockers in relation to Proteobacteria were linked to gut dysbiosis while no association was found between the microbiome and other three medications. In another population-based metagenomic analysis, there was positive correlation between the use of ACE inhibitors/ $\beta$-blockers and microbiota composition. Similarly, hypertensive rats administered ACE inhibitors captopril showed reduced gut dysbiosis including decreased intestinal permeability, fibrosis and improved villi length (5). Consequently, it can be deduced that drugs alter the gut microbiome, probably removing opportunistic and adverse pathogenic bacterial species (5). 


\section{Incorporating microbiome and drug interaction into clinical setting}

The influence of the microbiome on the effect and toxicity of drugs such as irinotecan (anti-cancer) and digoxin (cardiac drug) has been well recognized (49). In patients diagnosed with metastatic colorectal cancer receiving irinotecan (CPT-11), the build-up of metabolites of CPT-11 known as SN-38 glucuronide produced from microbial $\beta$-glucuronidases caused epithelial damage resulting into severe diarrhoea in some patients (31). In the application of shotgun metagenomics and targeted metabolomics, there was report of a characterized phylogenetically diverse group of bacterial $\beta$-glucuronidases and transporter proteins associated with high production of SN38 glucuronide as well as possible higher risk of irinotecan-dependent toxicity (50). Investigating metabolomics and metagenomic basis of diversification in drug metabolism employing exvivo drug incubations with human faecal samples may offer putative biomarkers of drug effectiveness or risk of treatment failure (49).

Metabolic phenotyping studies of microbiome-drug interaction using RNA/DNA high throughput sequencing with metabolomics have shown that the degree of complexity of gut microbiome related to drug metabolism differ between drugs $(50,51,52)$. The main impediment is the comprehension of what microbiome features identified through preclinical studies. Using model systems or human faecal samples as an alternative for the gut microbiome will translate into accurate surrogate endpoints for clinical studies. An example is the fact that the presence or absence of a candidate microbe or enzyme in a sequenced faecal sample may lack the ability to forecast drug metabolism (49). A way to overcome this barrier is to combine features using machine learning such as random forest method to detect the combinations of features most predictive of drug metabolism as this method can combine chemical and molecular features (49).

There are several clinical trials investigating the efficiency of probiotics to modify microbiome-dependent adverse drug responses. A study of the randomized double-blind design set up to explore the probiotic potential to minimize CPT-11 induced toxicity where patients were randomized into probiotic and placebo groups respectively showed that 39\% of participants in the probiotic group had grade 3 to 4 diarrhoea while $61 \%$ in placebo group had diarrhoea (53). Another way to reduce CPT-11 toxicity is the targeted inhibition of microbial enzymes that changes the inactive form of the drug to its active form. $\beta$ glucuronidase inhibitors from $E$. coli has been identified to significantly reduce CPT-11 induced toxicity in mice but not on the orthologous mammalian enzyme (31). A clinical trial taking the safety and efficiency of this method into cognizance in human population has the possibility of producing useful insight into the efficiency of targeted, small molecule modulators of specific microbiota functions (49).

\section{Conclusion:}

The evident involvement of microbiome especially of the gut community in drug metabolism has been established. Also, microbiome-drug interaction may be beneficial or detrimental, resulting in either treatment success or failure, which is largely dependent on factors such as the microbial enzymes, chemical composition of the candidate drug, host immunity and the complex relationship that exists with the microbiome. Despite the fact that several research studies have been conducted on this topic, there is still a large gap to the full understanding of the microbiome involvement in drug metabolism that will lead to improve treatment regimen in patients.

\section{References:}

1. Silbergeld, K. E. The Microbiome: Modulator of Pharmacological and Toxicological Exposures and Responses. Toxicol Pathol. 2017; 45 (1): 190-194.

2. Bisanz, E.J., Peter, S., Lindsey, M. P., Annamarie, E. B., and Peter, J. T. How to determine the role of the microbiome in drug disposition. Aspet J. 2018; DOI: $10.1124 /$ dmd.118.083402.

3. Kim, D. Special Section on Drug Metabolism and the Microbiome-Perspective Gut MicrobiotaMediated Drug-Antibiotic Interactions. Drug Metab Dispos. 2015; 43: 1581-1589.

4. Chankhamjon, P., Bahar, J., Jaime, L., Raphaella, H., Seema, C., and Mohamed, S. D. Systematic mapping of drug metabolism by the human gut microbiome. bioRxiv. 2019;

DOI: http://dx.doi.org/10.1101/538215.

5. Jama, H., David, M. K., and Franchine, Z. M. Population-Based Gut Microbiome Associations with Hypertension: The Need for More Detailed Phenotypes. Am Heart Ass J. 2018; 123: 11851187; doi:10.1161/CIRCRESAHA.118.313792.

6. Cussotto, S., Gerard, C., Timothy, G. D., and John, F. C. Psychotropics and the Microbiome: a Chamber of Secrets. Psychopharmacology. 2019; 236: 1411-1432.

7. Zhernakova, A., Kurilshikov, A., Bonder, M. J.., et al. Population-based metagenomics analysis reveals markers for gut microbiome composition and diversity. Science. 2016; 352: 565-569.

8. Ticinesi, A., Milani, C., Lauretani, F., et al.. Gut microbiota composition is associated with 
polypharmacy in elderly hospitalized patients. Sci Rep. 2017; 7: 11102.

9. Al-Salami, H., Butt, G., Fawcett, J. P., Tucker, I. G, Golocorbin-Kon S, and Mikov, M. Probiotic treatment reduces blood glucose levels and increases systemic absorption of gliclazide in diabetic rats. Eur J Drug Metab Pharmacok. 2008; 33:101-106.

10. Kim, J. K., Choi, M. S., Jeong, J. J., Lim, S. M., Kim, I. S., Yoo, H. H., and Kim, D. H. Effect of probiotics on pharmacokinetics of orally administered acetaminophen in mice. Drug Metab Dispos. 2018; 46:122-130

11. Kola, I., and Landis, J. Can the pharmaceutical industry reduce attrition rates? Nature Rev Drug Discov. 2004; 3: 711-715.

12. Artursson, P., Palm, K., and Luthman, K. Caco-2 monolayers in experimental and theoretical predictions of drug transport. Adv Drug Deliv Rev. 2001; 46: 27-43.

13. Kim, H. J., and Ingber, D. E. Gut-on-a-Chip microenvironment induces human intestinal cells to undergo villus differentiation. Integrative Biology. 2013; 5: 1130-1140.

14. Shah, P., Fritz, J. V., Glaab, E., et al. A microfluidics based in vitro model of the gastrointestinal human-microbe interface. Nature Commununications. 2016; 7: 11535.

15. Miller, D. S. Regulation of P-glycoprotein and other $A B C$ drug transporters at the blood-brain barrier. Trends Pharmacol Sci. 2010; 31: 246254.

16. Kramer, A., Green, J., Pollard, J., and Tugendreich, S. Causal analysis approaches in Ingenuity Pathway Analysis. Bioinformatics 2014; 30: 523-530.

17. Cory, T. J., He, H., Winchester, L. C., Kumar, S. and Fletcher, C. V. Alterations in glycoprotein expression and function between macrophage subsets. Pharmaceut Res. 2016; 33: 2713-2721.

18. Spanogiannopoulos, P., Bess E. N., Carmody R. N., and Turnbaugh, P. J. The microbial pharmacists within us: a metagenomic view of xenobiotic metabolism. Nature Rev Microbiol. 2016; 14: 273-287.

19. Maier, L., Pruteanu, M., Kuhn, M., et al. Extensive impact of non-antibiotic drugs on human gut bacteria. Nature. 2018; 555: 623-628.

20. Riley, M. A., and Lizotte-Waniewski, M. Population genomics and the bacterial species concept. Methods Molec Biol. 2009; 532: 367-377. Koppel, N., Bisanz J. E., Pandelia, M, Turnbaugh, P. J., and Balskus, E. P. Discovery and characterization of a prevalent human gut bacterial enzyme sufficient for the inactivation of a family of plant toxins. elife; 2018; 7: 28. doi: $10.7554 /$ eLife.33953.

22. Ding, W., Baumdicker, F., and Neher, R. A. panX: pan-genome analysis and exploration. Nucl Acids Res. 2018; 46: e5-e5.

23. McCabe, M., Sane, R. S., Keith-Luzzi, M., et al. Defining the role of gut bacteria in the metabolism of deleobuvir: in vitro and in vivo studies. Drug Metabolism and Disposition. 2015; 43: 16121618.

24. Yeo, H. K., Hyun, Y., Jang, S., Han, M. J., Lee, Y. S., and Kim, D. Development of fecal microbia enzyme mix for mutagenicity assay of natural products. J Microbiol Biotechnol. 2012; 22: 838848.

25. Yoo, D., Kim, I. S., Van Le, T. K., Jung, I., Yoo, H. H., and Kim, D. Gut microbiota mediated drug interactions between lovastatin and antibiotics. Drug Metab Dispos. 2014; 42: 1508-1513.

26. Gadaleta, R. M., Cariello, M., Sabbà, C., and Moschetta, A. Tissue-specific actions of FXR in metabolism and cancer. Biochim Biophys Acta. 2015; 1851: 30-39.

27. Swanson, H. I. Drug Metabolism by the Host and Gut Microbiota: A Partnership or Rivalry? Drug Metab Dispos. 2015; 43 (10): 1499-1504.

28. Kwok, C. S., Arthur, A. K., Anibueze, C. I., Singh, S. Cavallazzi, R., and Loke, Y. K. Risk of Clostridium difficile infection with acid suppressing drugs and antibiotics: meta-analysis. Am J Gastroenterol. 2012; 107: 1011-1019.

29. Carmody, R. N., and Turnbaugh, P. J. Hostmicrobial interactions in the metabolism of therapeutic and diet-derived xenobiotics. J Clin Investig. 2014; 124: 4173-4181.

30. Laparra, J. M., and Sanz, Y. Interactions of gut microbiota with functional food components and nutraceuticals. Pharmacol Res. 2010; 61 (3): 219-225.

31. Wallace, B. D., Wang, H., Lane, K. T., et al. Alleviating cancer drug toxicity by inhibiting a bacterial enzyme. Science. 2010; 330: 831-835.

32. Klaassen, C. D, and Cui, J. Y. Mechanisms of how the intestinal microbiota alters the effects of drugs and bile acids. Drug Metab Dispos. 2015; 43: 1505-1521.

33. Prosser, G. A., Copp, J. N., Syddall, S. P., et al. Discovery and evaluation of Escherichia coli nitroreductases that activate the anti-cancer prodrug CB1954. Biochem Pharmacol 2010; 79: 678-687.

34. Roldán, M. D., Pérez-Reinado, E., Castillo, F., and Moreno-Vivián, C. Reduction of polynitroaromatic compounds: the bacterial nitroreductases. Federation of European Microbiological Societies Microbiology Review. 2008; 32: 474-500.

35. Li, Y., Xu, J., Lai, W. G., Whitcher-Johnstone, A., and Tweedie, D. J. Metabolic switching of BILR 355 in the presence of ritonavir. II. Uncovering novel contributions by gut bacteria and aldehyde oxidase. Drug Metab Dispos. 2012; 40: 11301137

36. Sweeny, D. J., Li, W., Clough J., et al. Metabolism of fostamatinib, the oral methylene phosphate prodrug of the spleen tyrosine kinase inhibitor R406 in humans: contribution of hepatic and gut bacterial processes to the overall biotransformation. Drug MetabDispos. 2010; 38: $1166-1176$

37. Aura, A. M., Mattila, I., Hyötyläinen, T., et al. Drug metabolome of the simvastatin formed by human intestinal microbiota in vitro. Molecular Biosystem. 2011; 7: 437-446.

38. Rubin, D. C., Alava, P., Zekker, I., Du Laing, G., and Van de Wiele, T. Arsenic thiolation and the role of sulfate-reducing bacteria from the human intestinal tract. Environmental Health Perspectives. 2014; 122: 817-822.

39. Sowada, J., Schmalenberger, A., Ebner, I., Luch, A., and Tralau, T. Degradation of benzo[a] pyrene by bacterial isolates from human skin. Federation of European Microbiological Societies Microbiology Ecology. 2014; 88: 129-139.

40. Tripathi, A., Debelius, J., Brenner, D. A., Karin, M., Loomba, R., Schnabl, B., and Knight, R. The gut-liver axis and the intersection with the microbiome. Nature Rev Gastroenterol Hepatol. 2018; 11(815): 397-411.

41. Selwyn, F. P., Cui, J. Y., and Klaassen, C. D. RNASeq quantification of hepatic drug processing genes in germ-free mice. Drug Metab Dispos. 2015; 43: 1572-1580.

42. Panebianco, C., Andruili, A., and Paziienza, V. Pharmacomicrobiomics: exploiting the drugmicrobiota interactions in anticancer therapies. Microbiome. 2018; 6: 92. https://doi.org/10.1186/s40168-018-0483-7. 
43. Mikó, E., Kovacs, T., Sebo, E., Tóth, J., Csonka, T., Ujlaki, G., Sipos, A., Szabo, J., Méhes, G., and Bai, P. Microbiome-Microbial Metabolome-Cancer Cell Interactions in Breast Cancer-Familiar, but Unexplored. Cells. 2019; 8: 293. doi: $10.3390 /$ cells 8040293

44. Voorde, J. V., Sabuncuoglu, S., Noppen, S., Hofer, A., Ranjbarian, F., Fieuws, S., Balzarini, J., and Liekens, S. Nucleoside-catabolizing enzymes in mycoplasma infected tumor cell cultures compromise the cytostatic activity of the anticancer drug gemcitabine. J Biol Chemistr. 2014; 289 (19): 13054-13065.

45. Lehouritis, P., Cummins, J., Stanton, M., et al. Local bacteria affect the efficacy of chemotherapeutic drugs. Sci Rep. 2015; 5: 14554.

46. Geller, L.T., Barzily-Rokni, M., Danino, T., et al. Potential role of intratumor bacteria in mediating tumor resistance to the chemotherapeutic drug gemcitabine. Science. 2017; 357 (6356): 11561160.

47. Gui, Q. F., Lu, H. F., Zhang, C. X., Xu, Z. R., and Yang, Y. H. Well-balanced commensal microbiota contributes to anti-cancer response in a lung cancer mouse model. Genet Mol Res. 2015; 14 (2): 5642-5651. doi: 10.4238/2015.
48. Viaud, S., Saccheri, F., Mignot, G., et al. The intestinal microbiota modulates the anticancer immune effects of cyclophosphamide. Science. 2013; 342 (6161): 971-976.

49. Guthrie, L., and Kelly, L. Bringing microbiomedrug interaction research into the clinic. EBioMedicine. 2019; 44: 708-715.

50. Guthrie, L., Gupta, S., Daily, J., and Kelly, L. Human microbiome signatures of differential colorectal cancer drug metabolism. npj Biofilms Microbiomes. 2017; 3 (27): 709-713

51. Clayton, T. A., Baker, D., Lindon, J. C., Everett, J. R., and Nicholson, J. K. Pharmacometabonomic identification of a significant host-microbiome metabolic interaction affecting human drug metabolism. Proceedings Nat Acad Sci USA. 2009; 106: 14728-14733.

52. Haiser, H. J., Gootenberg, D. B., Chatman, K., Sirasani, G., Balskus, E. P., and Turnbaugh, P. J. Predicting and manipulating cardiac drug inactivation by the human gut bacterium Eggerthella lenta. Science. 2013; 341: 295-298.

53. Mego, M., Chovanec, J., Vochyanova-Andrezalova, I., et al. Prevention of irinotecan induced diarrhea by probiotics: a randomized double blind, placebo controlled pilot study. Complement Ther Med. 2015; 23: 356-362. 